\title{
A Dynamic Model of Altruistically-Motivated Transfers
}

\author{
Daniel Barczyk*and Matthias Kredler ${ }^{\dagger}$
}

June 14, 2008

\section{PRELIMINARY! DO NOT CITE.}

\begin{abstract}
Inter-vivos transfers constitute a quantitatively important mechanism of insurance, but their timing and influence on savings decisions are poorly understood. We develop a dynamic theory of voluntary transfers in a Markov-perfect setting with two infinitely-lived agents who are imperfectly altruistic. The agents' savings decisions are not Pareto-efficient since agents do not want to induce the other person to overconsume on their expense. As in the well-known static model of altruism, transfers are positively related to the donor's wealth and negatively related to the recipient's assets. The predictions of our model differ from the static framework in that the donor conditions on recipient's assets and income in distinct ways. A tentative result is that transfers only flow when the recipient's borrowing constraint binds, which is in line with stylized facts from the empirical literature. Unlike in other dynamic theories of partial insurance, agents continue to receive transfers even when they are not able to reciprocate them in the future.
\end{abstract}

\footnotetext{
*Department of Economics, New York University, db1395@nyu.edu

${ }^{\dagger}$ Department of Economics, Universidad Carlos Tercero (Madrid), mkredler@gmail.com
} 


\section{Introduction}

There are many economic situation in which inter-vivos transfers flow - potentially both ways - between two agents who are altruistically linked. For example, data from the Health and Retirement Study in the U.S. show that many people above age 50 make large monetary gifts to their children, whereas others - especially those in poor health - receive substantial transfers in both time and money from their children. ${ }^{1}$ Cox \& Raines (1985) showed that intervivos transfers are of a larger magnitude than bequests. Also paying for a child's college education can constitute a sizeable transfer, especially in Anglo-Saxon nations. Another prime example for arguably altruistically-motivated transfers are remittances by migrant workers to their families in developing countries, which have been shown to play an economically significant role.

Many static models for transfers in the tradition of Becker (1974) have been analyzed in the literature ${ }^{2}$, but there is a conspicuous lack of dynamic models. Therefore, the timing of transfers and their influence on savings decision is poorly understood. Our goal is to fill what we feel is a gap in the theoretical literature by providing dynamic theory of transfers with altruism. Moreover, we hope that our model will be used as a building block for models in the partial insurance literature where modeling the family is of importance. The lack of a dynamic theory of two-sided altruism makes it hard to build models for a host of issues such as long-term care, child care, bequests and education decisions; we hope that our model will aid the development of such theories. for dynamic models that are concerned with altruistically linked economic agents. In recent decades, panel data sets such as the Health and Retirement Study (HRS) and the Panel Study of Income Dynamics (PSID) have become available, which contain information on both monetary and non-monetary transfers between households and the economic situation of the involved parties.

Since savings decisions are at the heart of a great deal of economic issues related to inter-vivos transfers, a dynamic theory is needed. Policies concerning pension systems, long-term care and welfare payments all have important repercussions on savings decisions and are intertwined with the transfer decisions people make in informal-insurance networks like families. The crowding-out of informal transfers by government programs has been analyzed at lenght in static altruistic frameworks ${ }^{3}$, but there is much less work in a dynamic context.

The model we present in this paper is inhabited by two infinitely-lived agents who are linked to each other by mutual imperfect altruism. One-sided altruism, perfect altruism and egoistic preferences are all nested in this general setting. ${ }^{4}$

\footnotetext{
${ }^{1}$ See, for example, Brown (2007).

${ }^{2}$ See the short literature review below.

${ }^{3}$ See the surveys by Laitner (1997) and Laferrere \& Wolff (2006).

${ }^{4}$ Following Laitner's (1997) survey, throughout the paper we will refer to one-sided altruism as the following concept: Person A's ranking of allocations is influenced by person B's consumption - in a static setting, A may rank allocations according to a utility function $u\left(c_{A}\right)+\alpha u\left(c_{B}\right)$. Person B's ranking of allocations, however, is not affected by person A's consumption - B's preferences may be represented by utility $u\left(c_{B}\right)$ in this example. For twosided altruism, this dependence is mutual; B would have utility $u\left(c_{B}\right)+\alpha^{\prime} u\left(c_{A}\right)$, for example.
} 
Agents decide about consumption and savings in a riskless asset and can make a non-negative transfer to the other agent in each instant. We characterize the Markov-perfect equilibrium; agents can only condition their strategy on current states (both agents' assets and incomes), but not on past actions.

We find that as in the static model of altruism, transfers are positively linked to the donor's resources and negatively linked to the recipient's resources. One feature of our model is that transfers are especially likely to occur when one agent is borrowing-constrained, which is in line with empirical results (see Cox \& Jappelli, 1990 and Cox, 1990). When altruism is imperfect, agents' savings decisions are distorted with respect to the optimum that a benevolent family ruler would choose. Both transfer donors and recipients are led to overconsume due their strategic-altruistic considerations. Soon-to-be recipients of transfers fail to fully internalize the burden of transfers on donors; potential donors don't want to induce poorer family members to act imprudently by building up too many assets and thus creating expectations of large transfers. Almost ironically, efficiency is restored when one agent becomes dependent on the other's transfers forever - then, an effective family ruler is in charge in the person of the donor and his/her preferred allocation is implemented.

There is a large literature on one-sided altruism, starting with the now classical papers of Becker (1974) and Barro (1974). Becker develops a theory of altruism; Barro studies an overlapping-generations (OLG) economy with perfectly altruistic dynasties. Probably due to technical difficulties, the altruism literature has so far largely restricted itself to the case where the interaction between the individuals lasts for at most one period; examples are Laitner (1988) and more recently Fernandes (2008). A notable exception is Laitner (1992), who studies an OLG economy where children and parents co-exist for a finite number of periods $T$. However, in his setting altruism is perfect, which increases tractability substantially, as Laitner notes. Altonji, Hayashi \& Kotlikoff (1997) and McGarry (2006) test the hypothesis of altruism on transfer data based on predictions from a two-period model with one-sided altruism, but stop short of specifying a fully dynamic model. ${ }^{5}$

Our theory differs from other dynamic theories of partial insurance in that empoverished agents continue to receive transfers even when they have never transfered anything to the donor in the past and are unable to ever reciprocate in the future. Consider, for example, the theories of risk-sharing under limited commitment (Kocherlakota, 1996), exogenously incomplete markets ${ }^{6}$ and costs of state verification (Townsend, 1979): In all of these frameworks, since agents are ultimately selfish, one would never observe transfers to agents who are unable to reciprocate. In reality, however, we do observe such behavior, for example

We will refer to perfect altruism as the concept that person A is just as concerned about his own consumption as about person B's consumption, for example having a utility function $u\left(c_{A}\right)+u\left(c_{B}\right)$. Imperfect altruism is the obvious counterpart, represented for example by $u\left(c_{A}\right)+\alpha u\left(c_{B}\right)$ for $0<\alpha<1$.

${ }^{5}$ See also the surveys by Laitner (1997) and Laferrere \& Wolff (2006) for more references on both one-sided and two-sided altruism.

${ }^{6}$ By "exogenously incomplete markets" we mean economies in which only a pre-specified class of assets is traded. 
towards people with terminal diseases or disabilities.

On the technical side, our paper makes the following contributions: The continuous-time framework enables us to smooth out the non-concavity in the value function that arise in altruism models in discrete time; this is similar to Laitner (1988), who achieves the smoothing using lotteries. Furthermore, the transition to the limit in the spacing between decisions allows us to abstract from certain instantaneous strategic considerations because they are of second order, which greatly facilitates the analysis and computational exercises. Finally, the problem's Euler equations illustrate compactly how altruism introduce distortions into agents' savings decisions with respect to the Pareto-optimal allocation that were described before.

The remainder of the paper is structured as follows: In section 2, we briefly review the empirical facts on inter-vivos transfers. Section 3 presents our model in a general framework without uncertainty. Section 4 states the corresponding planner's problem and shows why the equilibrium from section 3 is in general not Pareto-optimal. Section 5 illustrates the model in a symmetric example with log-preferences and homogeneity in wealth and section 6 discusses an example with uncertainty in the income process. Section 7 concludes.

\section{Empirical facts}

Much of the empirical literature has focused on three types of intergenerational transfers: co-residence, time assistance and financial transfers. While our model is capable of accomodating a variety of transfers, it is most naturally interpreted when considering transfers in terms of money. In the past 20 years researchers in economics and sociology have uncovered empirical magnitudes and patterns regarding financial transfers within the family, mainly focusing on parents and their children.

One of the more surprising facts discovered earlier in the literature is that inter-vivos transfers are relatively large compared to bequests. Cox \& Raines (1985), using data from the 1979 President's Comission on Pension Policy survey, document that inter-vivos transfers are three times as large as inheritances. Gale \& Scholz (1994) provide additional evidence that transfers are large relative to bequests. They estimate an annual flow of cash transfers of $\$ 63$ billion (in 1986 dollars), whereas $\$ 105$ billion was transferred through bequests. Considering that bequests are made just once, the annual transfer amount is rather large.

Inter-vivos transfers are fairly common in the U.S., with most of the financial transfers flowing downward. Soldo \& Hill (1995) find that in the Health and Retirement Study about $40 \%$ gave financial assistance to a child of at least $\$ 500$. The median amount given to a child is $\$ 1,650$. Additionally, about $11 \%$ of the respondents of this survey provide transfers to their parents. Conditioning on married couples with both living parents and children indicates a median amount given to a child of $\$ 3,300$ and a median amount given to a parent of $\$ 1,200$. Similarly, McGarry \& Schoeni (1995) document that roughly $30 \%$ of 
parents in their fifties transfer cash of at least $\$ 500$ to their children, while $9 \%$ provide transfers to at least one parent. They find average magnitudes of $\$ 3,500$ for each child and $\$ 2,100$ for each parent, conditional on giving.

Lastly, while bequest are usually distributed equally among children, intervivos transfers are targeted toward liquidity-constrained children. Cox (1987) shows that the recipients are usually of low income, having a mean income of about $\$ 13,000$, and receiving a mean transfer amount of approximately $\$ 800$. More recently Berry (2008), using the Health and Retirement Study, finds that inter-vivos financial assistance goes to the economically most disadvantaged children. In his analysis he conditions on adult children defined as aged 18 or over and not living with parents. In families with multiple children, only $7 \%$ of donors give similar amounts to adult children. Economic characteristics which make it significantly more likely to become a recipient are working part time, being unemployed and not owning a home.

\section{Model}

\subsection{Setting}

Time $t$ is continuous. There are two agents in the economy who are infinitelylived. We will denote variables for the first agent, whom we will refer to as "she", as plain lower-case letters, e.g. $c_{t}$. Variables referring to the second agent, whom we we will call "he", are denoted with prime-superscripts, e.g. $c_{t}^{\prime}$. Both agents can hold a non-negative amount $k_{t}$ in a riskless asset that pays a a time-invariant rate of interest $r$. They receive a deterministic time-invariant income flow of $y$ and $y^{\prime}$, respectively.

In each instant of time, agents choose a consumption rate $c_{t} \geq 0$ and a nonnegative transfer rate $g_{t}$ to the other agent ( $g$ stands for "gift"), so that their assets evolve according to

$$
\begin{aligned}
& \dot{k}_{t}=r k_{t}+y-c_{t}-g_{t}+g_{t}^{\prime} \\
& \dot{k}_{t}^{\prime}=r k_{t}^{\prime}+y^{\prime}-c_{t}^{\prime}-g_{t}^{\prime}+g_{t},
\end{aligned}
$$

where dots denote the time-derivative of a variable. There is a no-borrowing constraint for both agents; when $k_{t}=0$, we must have that she does not spend more than she receives, i.e. $c_{t}+g_{t} \leq y+g_{t}^{\prime}$ (and equivalently form him, of course).

She ranks allocations using the criterion

$$
V_{0}=\int_{0}^{\infty} e^{-\rho t}\left[u\left(c_{t}\right)+\alpha u\left(c_{t}^{\prime}\right)\right] d t
$$

where $\rho>0$ is the discount rate and $0 \leq \alpha \leq 1$ the parameter which measures the intensity of altruism. ${ }^{7}$ He is a mirror-symmetric copy of her, but might

\footnotetext{
${ }^{7}$ With this linearly separable formulation of altruistic preferences we are in line with the bulk of the literature.
} 
have a different altruism parameter $0 \leq \alpha^{\prime} \leq 1$ than she has. His criterion for ranking allocations is

$$
W_{0}=\int_{0}^{\infty} e^{-\rho t}\left[u\left(c_{t}^{\prime}\right)+\alpha^{\prime} u\left(c_{t}\right)\right] d t .
$$

We assume that the agents do not differ in their discount rates and the form of the "primitive" utility function $u(\cdot)$.

The following special cases are of interest: When $\alpha=\alpha^{\prime}=0$, the problem collapses to two "selfish" savings problems which are independent of each other. When $\alpha>\alpha^{\prime}=0$, we speak of one-sided altruism of her towards him. In the case $\alpha=\alpha^{\prime}=1$, we speak of perfect two-sided altruism.

We define a Markov-perfect equilibrium as two consumption functions $c\left(k, k^{\prime}\right)$ and $c^{\prime}\left(k, k^{\prime}\right)$ and two transfer functions $g\left(k, k^{\prime}\right)$ and $g^{\prime}\left(k, k^{\prime}\right)$ which satisfy the following requirements:

- Given his policy $c^{\prime}\left(k, k^{\prime}\right)$ and $g^{\prime}\left(k, k^{\prime}\right)$, her policy $c\left(k, k^{\prime}\right)$ and $g\left(k, k^{\prime}\right)$ maximizes $V_{0}$ for any initial $\left(k_{0}, k_{0}^{\prime}\right)$ (and vice versa for him).

- The consumption and transfer strategies satisfy the no-borrowing constraints.

- $k_{t}$ and $k_{t}^{\prime}$ follow the laws of motion (1) and (2)

In the following, we will often write $c_{t}=c\left(k_{t}, k_{t}^{\prime}\right)$ and $g_{t}=g\left(k_{t}, k_{t}^{\prime}\right)$, where it is understood that we plug in the capital stocks determined by the previous policies $c\left(k_{s}, k_{s}^{\prime}\right)$ etc. for $0 \leq s \leq t$.

The strategy of the analysis will be the following: First, we discuss the firstorder conditions in regions where no transfers flow (subsection 3.2). We then characterize the optimal transfer decision when no agent is constrained and derive conditions under which no transfers flow (subsection 3.3). In subsection 3.4, we proceed to derive the optimal transfers when one agent is constrained and prove that given this "terminal condition", it is optimal never to give transfers when the recipient still owns some assets. In the sections 5 and 6 , these principles are illustrated in two examples.

\subsection{No-transfer region: Euler equations}

Consider an open set of $\mathbb{R}^{2}$ in which $k>0, k^{\prime}>0$ and transfers are zero, i.e. $g=g^{\prime}=0$. Suppose that we know her value function $V\left(k, k^{\prime}\right)$. When we take his policy $c^{\prime}\left(k, k^{\prime}\right)$ as given, her strategy over a short time interval $\Delta t$ must fulfill Bellman's principle:

$$
\begin{aligned}
V\left(k, k^{\prime}\right) & \simeq \max _{c}\left\{u(c) \Delta t+\alpha u\left(c^{\prime}\right) \Delta t+e^{-\rho \Delta t} V\left(k+\Delta k, k^{\prime}+\Delta k^{\prime}\right)\right\} \\
\Delta k & =(r k+y-c) \Delta t \\
\Delta k^{\prime} & =\left(r k^{\prime}+y^{\prime}-c^{\prime}\right) \Delta t
\end{aligned}
$$


This equation says that the value function at a point $\left(k, k^{\prime}\right)$ is approximately given by the flow value from her consumption and a fraction of his consumption value over the interval $\Delta t$ plus the discounted value that she has after that interval. She has to trade off immediate gains from current consumption, which are captured in $u(c)$, against the gains from saving, which are encoded in the behavior of the value function in the $k$-direction, as we will see in a moment.

Now, take a first-order Taylor expansion of the expression $e^{-\rho \Delta t} V(\cdot, \cdot)$ in $\Delta t$ around the point $\Delta t=0$ and go to the limit $\Delta t \rightarrow 0$ to obtain the HamiltonJacobi-Bellman equation (HJB):

$$
0=-\rho V\left(k, k^{\prime}\right)+\dot{k}^{\prime} V_{k^{\prime}}\left(k, k^{\prime}\right)+\max _{c}\left\{u(c)+\alpha u\left(c^{\prime}\right)+(r k-c) V_{k}\left(k, k^{\prime}\right)\right\},
$$

where we denote partial derivatives by subscripts. Throughout the analysis, we will assume that both value functions are twice continuously differentiable. The first-order condition associated with the HJB is

$$
u^{\prime}\left[c\left(k, k^{\prime}\right)\right]=V_{k}\left(k, k^{\prime}\right),
$$

which says that the marginal utility of current consumption is set equal to the marginal value of saving in the optimum.

It is noteworthy that his consumption decision $c^{\prime}\left(k, k^{\prime}\right)$, which is present in the term $\dot{k}^{\prime}$, does not affect her optimal choice $c$. In game-theoretic terms we would say that her best-response function is a constant. This is a considerable simplification of the problem, which does not arise in the analogous discretetime problem; it occurs because immediate strategic considerations turn out to be of second order. To see why this is the case, consider the following first-order approximation of the marginal value of saving in a small neighborhood of the current state $\left(k, k^{\prime}\right)$ :

$$
\begin{aligned}
V_{k}\left(k+\Delta k, k^{\prime}+\Delta k^{\prime}\right)= & V_{k}\left(k, k^{\prime}\right)+V_{k k}\left(k, k^{\prime}\right)(r k+y-c) \Delta t+ \\
& +V_{k k^{\prime}}\left(r k^{\prime}+y^{\prime}-c^{\prime}\right) \Delta t+o(\Delta t)
\end{aligned}
$$

We see that as $\Delta t$ becomes small, the changes in the marginal value of saving over the planning period $\Delta t$ become negligible. So even when he chooses a very high $c^{\prime}$, we can still find $\Delta t$ small enough so that $V_{k}$ is (almost) constant over the planning horizon. It is therefore valid to get rid of the interaction effects between the consumption decisions. Economically speaking, an agent who reconsiders her savings decisions on a daily basis need not worry about the daily savings decisions of her counterpart, since the latter will not have a large impact on the other's assets - it is enough to keep an eye on the other's bank account to be sufficiently informed.

In order to learn more about the intertemporally optimal consumption plan, we will now study the Euler equations of this problem. Euler equations tell us how marginal utility of consumption changes over time - recall that in discrete time for a selfish agent, we have $u^{\prime}\left(c_{t}\right)=\beta R u^{\prime}\left(c_{t+1}\right)$, which is a difference equation in $c_{t}$. In continuous time, we can obtain an analogous differential 
equation from the HJB: First, let us focus on the selfish case by setting $\alpha=$ $\alpha^{\prime}=0$, where the HJB (3) reads

$$
0=u\left[c^{*}(k)\right]-\rho V(k)+\left[r k-c^{*}(k)\right] V_{k}(k) .
$$

Note that we got rid of the max-operator by substituting in the optimal policy $c^{*}(k)$ and removed the $k^{\prime}$-dimension since it does not matter. Now, take the derivative of this equation in $k$ to obtain

$$
0=\frac{d c(k)}{d k}\left[u^{\prime}(c)-V_{k}(k)\right]-\rho V_{k}+r V_{k}+\dot{k} V_{k k}(k),
$$

where we write $\dot{k}=r k-c^{*}(k)$ for the law of motion resulting from the optimal consumption decision. In brackets, we recognize the first-order condition (4), which evaluates to zero. Also, notice that the term $\dot{k} V_{k k}$ tells us something about the behavior of $V_{k}$ over time along the optimal path. We have:

$$
\frac{d}{d t} V_{k}\left(k_{t}\right)=\left(V_{k}\right)_{k} \dot{k}_{t}=V_{k k} \dot{k}_{t} .
$$

Thus we obtain the following Euler equation for a selfish agent:

$$
\frac{d}{d t} V_{k}\left(k_{t}\right)=(\rho-r) V_{k}=\frac{d}{d t} u^{\prime}\left(c_{t}\right),
$$

where the last equality follows from the first-order condition (4). This equation tells us something familiar: If $\rho>r$, the agent is impatient compared to other market participants her marginal utility grows at rate $(\rho-r)$ over time, which implies a decreasing consumption path. If $\rho=r$, then marginal utility is timeinvariant (or follows a martingale, in the stochastic case). For $\rho<r$, the agent is more patient than other market participants and his consumption is increasing over time.

When carrying out the same steps as in the selfish problem for the case with altruism (i.e. $\alpha>0$ and $\alpha^{\prime}>0$ ), we obtain

$$
0=(r-\rho) V_{k}+\dot{k} V_{k k}+\dot{k}^{\prime} V_{k k^{\prime}}+\left[\alpha u^{\prime}\left(c^{\prime}\right)-V_{k^{\prime}}\right] \frac{d c^{\prime}}{d k} .
$$

As in the selfish case, we will again try to see how $V_{k}$ develops along the equilibrium path. To do this, parameterize $V_{k}$ with $t$ by writing $V\left[k(t), k^{\prime}(t)\right]$, then use the chain rule of differentiation to find that

$$
\frac{d V_{k}}{d t}=\left(V_{k}\right)_{k} \dot{k}+\left(V_{k}\right)_{k^{\prime}} \dot{k}^{\prime}=(\rho-r) V_{k}+\underbrace{\left[V_{k^{\prime}}-\alpha u^{\prime}\left(c^{\prime}\right)\right]}_{\equiv \lambda\left(k_{t}, k_{t}^{\prime}\right)} \frac{d c^{\prime}}{d k} .
$$

The intuition here is the following: The $\operatorname{term}(\rho-r) V_{k}$ tells us that marginal utility increases at rate $(\rho-r)$ along the optimal consumption path, which is the same as in the selfish setting. The second term is related to altruistic/strategic considerations: When she saves one unit more over some interval of time, her 
capital stock will increase and there will be a (usually positive ${ }^{8}$ ) effect on his consumption which is captured in the term $d c^{\prime} / d k$. This increase in $c^{\prime}$ gives an immediate gain $\alpha u^{\prime}\left(c^{\prime}\right)$ to her since she feels happy for him; this is an additional incentive to save, so it enters the equation with the same sign as $r$ does. However, his increased consumption also means that he saves less, which has negative effects on her (through decreased future consumption and decreased future transfers) that are captured in the term $V_{k^{\prime}}$. These enter with the same sign as $\rho$, so they act as a disincentive to save.

We will now see that the disincentive to save will dominate under quite general conditions, i.e. we will argue that $\lambda=V_{k^{\prime}}-\alpha u^{\prime}\left(c^{\prime}\right)=V_{k^{\prime}}-\alpha W_{k^{\prime}}>0$. Let us assume that future consumption is a differentiable function of the current state, i.e. there exists the following "impulse response": $d c_{s} / d k_{t}$ exists for all $s>t$ (TO PROVE IN APPENDIX). Then, writing out the value functions as integrals over future consumption and taking the derivative below the integral sign, we find that

$$
\begin{aligned}
\frac{d V\left(k_{t}, k_{t}^{\prime}\right)}{d k_{t}^{\prime}} & =\int_{0}^{\infty} e^{-\rho(s-t)}\left[u^{\prime}\left(c_{s}\right) \frac{d c_{s}}{d k_{t}^{\prime}}+\alpha u^{\prime}\left(c_{s}^{\prime}\right) \frac{d c_{s}^{\prime}}{d k_{t}^{\prime}}\right] d s \\
\alpha \frac{d W\left(k_{t}, k_{t}^{\prime}\right)}{d k_{t}^{\prime}} & =\alpha \int_{0}^{\infty} e^{-\rho(s-t)}\left[u^{\prime}\left(c_{s}^{\prime}\right) \frac{d c_{s}^{\prime}}{d k_{t}^{\prime}}+\alpha u^{\prime}\left(c_{s}\right) \frac{d c_{s}}{d k_{t}^{\prime}}\right] d s,
\end{aligned}
$$

from which it follows that

$$
\lambda_{t}=\frac{d V\left(k_{t}, k_{t}^{\prime}\right)}{d k_{t}^{\prime}}-\alpha \frac{d W\left(k_{t}, k_{t}^{\prime}\right)}{d k_{t}^{\prime}}=\left(1-\alpha^{2}\right) \int_{t}^{\infty} e^{-\rho(s-t)} u^{\prime}\left(c_{s}\right) \frac{d c_{s}}{d k_{t}^{\prime}} d s
$$

We expect that $\frac{d c_{s}}{d k_{t}^{t}}>0$ for all $s>t$, i.e. agent 1 tends to consume more at any point in the future if we let agent 2 start out at a higher wealth level. After all, higher wealth for agent 2 should mean that agent 2 will need fewer transfers from agent 1 in the future, or - if agent 2 ends up in the situation of the donor - agent 2 should be able to dole out larger transfers to agent 1.

We conclude that altruistic-strategic considerations induce an extra disincentive to save. Agents fear that excessive savings on their part induce the other agent to spend lavishly, relying on the larger resources of their counterpart. In the planner's problem in section 4 , we will see that this altruisticstrategic effect leads to inefficiency of the equilibrium allocation with respect to the Pareto-optimal allocation.

To complete our system of Euler equations, consider now the entire set of derivatives of the functions $V$ and $W$ in both the $k$-and $k^{\prime}$-direction. As for $V_{k^{\prime}}$, we can differentiate the HJB with respect to $k^{\prime}$ and then follow the same steps as before. For his value function $W$, we can then write down the same

\footnotetext{
${ }^{8}$ In the case where he is richer than she is, he will feel more confident about consuming more when she increases her wealth since it becomes less likely that he must help her out or later. If she is rich relative to him, he will know that there are more resources available that she can potentially provide to him in the form of transfers, which again tends to increase his consumption.
} 
expressions in a mirror-symmetric way to obtain the system:

$$
\begin{aligned}
\frac{d V_{k}}{d t} & =(\rho-r) V_{k}+\left(V_{k^{\prime}}-\alpha W_{k^{\prime}}\right) \frac{d c^{\prime}}{d k} \\
\frac{d V_{k^{\prime}}}{d t} & =(\rho-r) V_{k^{\prime}}+\left(V_{k^{\prime}}-\alpha W_{k^{\prime}}\right) \frac{d c^{\prime}}{d k^{\prime}} \\
\frac{d W_{k}}{d t} & =(\rho-r) W_{k}+\left(W_{k}-\alpha^{\prime} V_{k}\right) \frac{d c}{d k} \\
\frac{d W_{k^{\prime}}}{d t} & =(\rho-r) W_{k^{\prime}}+\underbrace{\left(W_{k}-\alpha^{\prime} V_{k}\right) \frac{d c}{d k^{\prime}}}_{\text {"altruistic-strategic distortions" }}
\end{aligned}
$$

We see that $V_{k^{\prime}}$ - just as $V_{k}$ before - grows at rate $(\rho-r)$ but is also affected by a term involving $\lambda$. In section 3.2 , we will see how the growth of $V_{k}$ is related to the growth of $V_{k^{\prime}}$. For him, of course, the differential equations are the analogon to hers.

Note that (6) is not an autonomous system of ordinary differential equations, i.e. we cannot take values $\left(V_{k}, V_{k^{\prime}}, W_{k}, W_{k^{\prime}}\right)$ at some final point $\left(k_{T}, k_{T}^{\prime}\right)$ and then solve the system (6) backward. ${ }^{9}$ This is impossible since the terms $d c_{i} / d k_{j}$ contain derivatives that go in directions pointing away from the equilibrium path. To see this, take (for example) the derivative of the first-order condition (4) in the $k$-direction:

$$
\frac{d c}{d k}=\frac{\left(V_{k}\right)_{k}}{u^{\prime \prime}(c)}
$$

which shows that we must be able to evaluate the derivative of $V_{k}$ in the $k$ direction. Therefore, the mere knowledge of $d V_{k} / d t=\dot{k}\left(V_{k}\right)_{k}+\dot{k}^{\prime}\left(V_{k}\right)_{k^{\prime}}$ is not sufficient for the ODE approach, since many values of $V_{k k}$ are conformable with a given value of $d V_{k} / d t$.

Economically speaking, this means that we need to know the behavior of consumption in other directions than just the equilibrium path (the direction $\left.\left(\dot{k}, \dot{k}^{\prime}\right)\right)$ in order to determine savings decisions. The agents take into account what would happen if they deviated from the equilibrium strategy, even if those deviations never happen in equilibrium. In order to make headway on the system (6), we need to solve for the equilibrium allocation also in a neighborhood

\footnotetext{
${ }^{9}$ In the selfish savings problem we can actually do this; there, indeed we can solve the Euler equation

$$
\frac{d}{d t}\left[u^{\prime}(c)\right]=\dot{k} V_{k k}=(\rho-r) u^{\prime}(c)
$$

backward in time without knowing what the optimal looks like off the optimal path. This ODE is autonomous, i.e. we do not need to know higher derivatives in order to solve for the optimal allocation. In PDE language, this is because the second derivative $V_{k k}$ enters the equation in such a way that we follow exactly the characteristic line (i.e. the equilibrium path). This is not the case in the system with altruism. It turns out that when we take derivatives of the equations in (6) again in order to find out something about the behavior of the second derivatives, we obtain a very complicated expression which seems untractable.
} 
of the equilibrium path, which will involve solving a partial differential equation (PDE) instead of a simpler ODE. ${ }^{10}$.

\subsection{Transfer decision}

So far, we have assumed that transfers are zero. Let us now consider the control problem including transfers. When taking as given his strategy $\left(c^{\prime}, g^{\prime}\right)$, Bellman's principle states the following for her optimal policy $(c, g)$ :

$$
\begin{aligned}
V\left(k, k^{\prime}\right) & \simeq \max _{c, g \geq 0}\left\{u(c) \Delta t+\alpha u\left(c^{\prime}\right) \Delta t+e^{-\rho \Delta t} V\left(k+\Delta k, k^{\prime}+\Delta k^{\prime}\right)\right\} \\
\Delta k & =\left(r k+y-c-g+g^{\prime}\right) \Delta t \\
\Delta k^{\prime} & =\left(r k^{\prime}+y^{\prime}-c^{\prime}-g^{\prime}+g\right) \Delta t
\end{aligned}
$$

When taking the limit $\Delta t \rightarrow 0$, we find the following HJB:

$$
\begin{aligned}
0= & \alpha u\left(c^{\prime}\right)-\rho V\left(k, k^{\prime}\right)+\left[r k+y-g^{\prime}\right] V_{k}\left(k, k^{\prime}\right)+\left[r k^{\prime}+y^{\prime}-c^{\prime}\right] V_{k^{\prime}}\left(k, k^{\prime}\right)+ \\
& +\max _{g \geq 0}\left\{g[\underbrace{V_{k^{\prime}}\left(k, k^{\prime}\right)-V_{k}\left(k, k^{\prime}\right)}_{\equiv \mu\left(k, k^{\prime}\right)}]\right\}+\max _{c}\left\{u(c)-c V_{k}\left(k, k^{\prime}\right)\right\}
\end{aligned}
$$

We see immediately that the transfer decision can be disentangled from the consumption decision in the limit. The intuition for this result is just as before: Consumption is a flow variable and does not affect stock variables significantly in the short run. Since the value function depends only on stock variables, we can neglect the effects of flow variables in the limit.

Another insight we glean from (7) is that the optimal transfer policy is of the bang-bang type: If the term $\mu \equiv\left(V_{k^{\prime}}-V_{k}\right)$, which we will refer to as the "transfer motive", is positive, then the agent wants to choose $g$ as large as possible. In fact, if the agent is allowed to make transfers that are mass points, she will choose to follow the vector $(-1,1)$ in $\left(k, k^{\prime}\right)$-space as long as the directional derivative $V_{k^{\prime}}-V_{k}=\mu$ is positive. If the transfer motive $\mu$ is negative, then she will choose the lower limit $g=0$; after she cannot force him to give transfers to her.

As mentioned before, we will adopt the following strategy: First, we guess that transfers only flow when the recipient is broke and determine the transfer motive at the limit when $k^{\prime} \rightarrow 0$ (see section 3.4). Then, we use a linear combination of the first and second equation in the system (6) to see how the transfer motive evolves over time:

$$
\frac{d}{d t} \mu=\frac{d}{d t}\left(V_{k^{\prime}}-V_{k}\right)=\frac{d}{d t} V_{k^{\prime}}-\frac{d}{d t} V_{k}=(\rho-r) \mu+\lambda(\underbrace{\frac{d c^{\prime}}{d k^{\prime}}-\frac{d c^{\prime}}{d k}}_{=d c^{\prime} / d g})
$$

The first term on the right-hand side is as in the selfish agent's Euler equation: The transfer motive increases at a rate $(\rho-r)$ when neglecting altruistic-strategic

\footnotetext{
${ }^{10}$ In section, though, we make a simplifying homogeneity assumptionm which allows us to return to an ODE approach.
} 
considerations. Let us now turn to the second term: $d c^{\prime} / d g \equiv d c^{\prime} / d k^{\prime}-d c^{\prime} / d k$ is the marginal effect of a small transfer by her on his consumption, which we expect to be positive. This multiplies $\lambda>0$, which we are familiar with from the Euler equations.

Let us now integrate up equation (8) from $t$ until some future $T$, which we will later take to be the moment at which he goes broke:

$$
\mu_{t}=-\left.\int_{t}^{T} e^{-(\rho-r)(s-t)} \lambda_{s} \frac{d c^{\prime}}{d g}\right|_{s} d s+e^{-(\rho-r)(T-t)} \mu_{T}
$$

If $\mu_{T}<0$, i.e. there is no desire to give transfers at $T$, than we can conclude that also $\mu_{t}<0$ for all $t<T$ if $\frac{d c^{\prime}}{d g}>0$ (recall that the argument from section 3.2 suggested that we have $\lambda_{s}>0$ ). Economically speaking, if already at $T$ transfers are not desirable, then there are only more arguments against transfers piling up as we move backward in time from $T$. If she gave a unit of cash to him before he goes bankrupt, then he would increase consumption in a way that she would not approve of $(\lambda>0)$.

\subsection{Region where one player is bankrupt}

We will now consider the case where one player, say she, is bankrupt. Given his transfer and consumption decision, her (now constrained) problem is given by the HJB

$$
0=-\rho V+\dot{k}^{\prime} V_{k^{\prime}}+\max _{0 \leq c \leq y+g^{\prime}}\left\{u(c)+\alpha u\left(c^{\prime}\right)+\left[y+g^{\prime}-c\right] V_{k}\right\} .
$$

Notice that now we will obtain corner solutions if $u^{\prime}\left(y+g^{\prime}\right)>V_{k}$, i.e. the marginal utility of consuming the last unit of the current inflow of assets is higher than the marginal value of saving. We will refer to this situation as her being constrained. When she is constrained, her best-response function is $c\left(c^{\prime}, g^{\prime} ; 0, k^{\prime}\right)=g^{\prime}+y$. He, taking this into account, will then make the transfer just to equalize the marginal returns on both margins (his and her consumption):

$$
\alpha^{\prime} u^{\prime}\left(y+g^{\prime}\right)=u^{\prime}\left(c^{\prime}\right)\left(=W_{k^{\prime}}\right)
$$

If the left-hand side was smaller than the right-hand side, he could certainly increase his payoff by re-allocating from his consumption to hers. Once he reaches the point where she ceases to be constrained, i.e. $u^{\prime}\left(y+g^{\prime}\right) \geq V_{k}$, she would start to save some of his transfer. In the following, we will proceed to show that it is not in his interest to do this.

Let us start assuming that we know the value function on the line where she is bankrupt $\left(k=0, k^{\prime}>0\right)$. We are looking for a solution to the PDEs which is continuously differentiable in both directions on the inside, i.e. for all points with $k>0, k^{\prime}>0$. Obviously, we will have continuity at the border, i.e. $V\left(\epsilon, k^{\prime}\right) \rightarrow V\left(0, k^{\prime}\right)$ when $\epsilon \rightarrow 0$.

It is also clear that for $k=0$, we have continuity of $V_{k}$ at any point $\left(0, k^{\prime}\right)$. Suppose that was not the case and we had $\lim _{\epsilon \rightarrow 0} V_{k}\left(\epsilon, k^{\prime}\right) \neq V_{k}\left(0, k^{\prime}\right)$. Then 
consider the hyperplane approximating $V$ in the point $\left(0, k^{\prime}\right)$ which is described by $V\left(0, k^{\prime}\right)+V_{k}^{\text {lim }}\left(0, k^{\prime}\right) \Delta k+V_{k^{\prime}}^{\text {lim }}\left(0, k^{\prime}\right) \Delta k^{\prime}$ where the superscript lim indicates that we use the limiting values of the derivatives coming from the inside. The line that is formed by the cut of this hyperplane with the $k=0$-plane is not equal to the line described by $V\left(0, k^{\prime}\right)+V_{k^{\prime}}\left(0, k^{\prime}\right) \Delta k^{\prime}$ by the contradicting assumption. But then $V$ would not be continuous in some small neighborhood of $\left(0, k^{\prime}\right)$, which contradicts the continuity of $V$.

From the continuity of the derivatives of the value function we can now derive some fundamental facts about consumption when $k \rightarrow 0$. First, note that by continuity of $W_{k^{\prime}}$ we must have that

$$
c^{\prime}\left(\epsilon, k^{\prime}\right) \rightarrow c_{1}^{\prime}\left(k^{\prime}\right) \quad \text { as } \epsilon \rightarrow 0
$$

since $W_{k^{\prime}}$ and hence $u^{\prime}\left(c^{\prime}\right)=W_{k^{\prime}}$ converge to the limit given by $W_{1}\left(k^{\prime}\right)$.

For the agent near bankruptcy matters are slightly more complicated. First, let us define

$$
c_{0}^{\lim }\left(k^{\prime}\right) \equiv \lim _{\epsilon \rightarrow 0} c\left(\epsilon, k^{\prime}\right) .
$$

This is well-defined since $u^{\prime}(c)=V_{k}$ and the continuity of $V_{k}$ guarantee continuity for $c\left(k, k^{\prime}\right)$ on the inside. Since the HJB is fulfilled everywhere on the inside, also for $k=0$ the limits of the respective terms must fulfill it, so we see that $c_{0}^{\text {lim }}$ must fulfill the following equation:

$$
0=u\left[c_{0}^{l i m}\left(k^{\prime}\right)\right]+\alpha u\left[c^{\prime}\left(0, k^{\prime}\right)\right]-\rho V\left(0, k^{\prime}\right)+\dot{k} V_{k}^{l i m}\left(0, k^{\prime}\right)+\dot{k}^{\prime} \lim \left(0, k^{\prime}\right) V_{k^{\prime}}\left(0, k^{\prime}\right)
$$

where we have already taken the limits for $V, V_{k^{\prime}}$ and $c^{\prime}$, which is legal by the arguments above. Notice that

$$
k^{l i m}\left(k^{\prime}\right)=\lim _{\epsilon \rightarrow 0} r \epsilon-c\left(\epsilon, k^{\prime}\right)=c_{0}^{l i m}\left(k^{\prime}\right),
$$

so we may write

$$
\begin{aligned}
-\left.\frac{d V}{d t}\right|_{\left(0, k^{\prime}\right)} & =-c_{0}^{l i m}\left(k^{\prime}\right) V_{k}^{l i m}\left(0, k^{\prime}\right)-\dot{k}^{\prime l i m}\left(0, k^{\prime}\right) V_{k^{\prime}}\left(0, k^{\prime}\right)= \\
& =u\left[c_{0}^{l i m}\left(k^{\prime}\right)\right]+\alpha u\left[c^{\prime}\left(0, k^{\prime}\right)\right]-\rho V\left(0, k^{\prime}\right)
\end{aligned}
$$

This equation is interpreted as follows: When fixing limiting consumption to some value $c_{0}^{\text {lim }}$, we may back out what the value function must have been a small amount of time before bankruptcy. The second line says that we must add flow utility in this instant and discount the value function since bankruptcy is postponed for a little. The right-hand side of the first line tells us in which direction in $\left(k, k^{\prime}\right)$-space we must move to read off this change in the value function. This allows us to obtain the derivative of $V$ in the $k$-direction, taking $c_{0}^{\lim }$ as given.

Note now, however, that again consumption is optimally chosen, and that we must have $u^{\prime}\left(c_{0}^{\text {lim }}\right)=V_{k}^{\text {lim }}$ if the agent behaves optimally. Plugging this into the above gives us

$$
u^{\prime}\left(c_{0}^{\lim }\right)=\frac{u\left(c_{0}^{\lim }\right)+\alpha u\left(c_{0}^{\prime}\right)-\rho V_{0}+\dot{k}^{\prime} V_{k^{\prime}}}{c_{0}^{\lim }}
$$


This equation says that the marginal benefit from consumption on the lefthand side must be equal to its marginal cost. This cost is that bankruptcy is hastened, which means that the agent obtains the flow value of bankruptcy instead of his own consumption for some time less. Also, if the other agent is saving, bankruptcy will be entered with a lower $k^{\prime}$ if bankruptcy is accelerated by high consumption.

Now that we know consumption for both agents, we can also back out the derivative of both value functions in the $k$-direction. For $V_{k}$ this is easy because we have $V_{k}\left(0, k^{\prime}\right)=u^{\prime}\left(c_{0}^{l i m}\right)$. For $W_{k}$, observe that the value function just before bankruptcy is given by current flow utility plus the discounted value of bankruptcy. So we may read off from the HJB how the value function changes in the $k^{\prime}$-direction, exploiting the fact that the value function is locally wellapproximated by a plane:

$$
\begin{aligned}
c_{0}^{\lim } W_{k}+\left(r k^{\prime}-c_{0}^{\prime}\right) W_{k^{\prime}} & =u\left(c_{0}^{\prime}\right)+\alpha u\left(c_{0}^{\lim }\right)-\rho W \quad \Rightarrow \\
W_{k} & =\frac{1}{c_{0}^{\lim }}\left[u\left(c_{0}^{\prime}\right)+\alpha u\left(c_{0}^{l i m}\right)-\rho W_{1}-\left(r k^{\prime}-c_{0}^{\prime}\right)\left(W_{1}\right)_{k^{\prime}}\right]
\end{aligned}
$$

\section{The planner's problem}

Given $k_{0}$ and $k_{0}^{\prime}$, the planner chooses optimal savings policies $k(t), k^{\prime}(t)$ and consumption policies $c(t), c^{\prime}(t)$ for $0 \leq t<\infty$ to maximize

$$
J=\int_{0}^{\infty} e^{-\rho t}[\underbrace{u\left(c_{t}\right)+\alpha u\left(c_{t}^{\prime}\right)}_{\equiv U_{t}}] d t-\eta \int_{0}^{\infty} e^{-\rho t}[\underbrace{u\left(c_{t}^{\prime}\right)+\alpha^{\prime} u\left(c_{t}\right)}_{\equiv U_{t}^{\prime}}] d t,
$$

This approach will yield all allocations on the Pareto frontier when we vary $\eta$.

Obviously, we can pool the single capital stocks together and define $K=$ $k+k^{\prime}$ as total resources of the family. It is also straighforward how the planner must allocate resources intratemporally in any instant: To equalize the two margins, the following must hold:

$$
\left.u^{\prime}\left(c_{t}\right)+\eta \alpha^{\prime} u^{\prime}\left(c_{t}^{\prime}\right)=\eta u^{\prime}\left(c_{t}^{\prime}\right)+\alpha u^{\prime}\left(c_{t}\right)\right] \quad \forall t,
$$

Plugging in yields

$$
u^{\prime}\left(c_{t}\right)=\frac{\eta+\alpha}{1+\alpha^{\prime} \eta} u^{\prime}\left(c_{t}^{\prime}\right) \quad \forall t
$$

This gives us $c_{t}^{\prime}$ as a function of $c_{t}$, and we can see that the problem has collapsed to a selfish savings problem with a different objective function; to see this, plug $c_{t}^{\prime}$ into the objective (9).

We also see from the intratemporal optimally condition (10) that efficiency holds when one agent is bankrupt. Recall that the donor sets $u^{\prime}(c)=\alpha u^{\prime}\left(c^{\prime}\right)$ to recover the Pareto weight that this implies for the bankruptcy situation:

$$
\alpha=\frac{\eta+\alpha}{1+\alpha^{\prime} \eta}
$$


Solving yields $\eta=0$, which means that the planner assigns zero weight to the bankrupt player. This makes sense since the allocation is in this case is effectively chosen by the rich agent in equilibrium. Also note that all paths starting from some $k>0, k^{\prime}>0$ that lead to bankruptcy cannot be efficient if they do not set $u^{\prime}\left(c_{t}\right)=\alpha u^{\prime}\left(c_{t}^{\prime}\right)$ - most likely not the case...

Intertemporally, we can see that the agent's selfish Euler equations must hold. If that was not the case, the family planner should definitely re-allocate resources in a way that yielded higher value to the agent without requiring more life-time wealth. This means that we must have

$$
\begin{aligned}
\frac{d}{d t} u^{\prime}(c) & =(\rho-r) u^{\prime}(c) \\
\frac{d}{d t} u^{\prime}\left(c^{\prime}\right) & =(\rho-r) u^{\prime}\left(c^{\prime}\right)
\end{aligned}
$$

When comparing these equations to the Euler equations of the equilibrium allocation in (6), we see that marginal utility of the agents in general grows faster in equilibrium than if a family ruler is in charge. This is due to the altruisticstrategic distortions that were discussed in section 3.2.

\section{A symmetric example with homogeneity in wealth}

This section demonstrates per example how the model can be solved. The setting is simple: Preferences are represented by a logarithmic utility function, there is no uncertainty, households are endowed with some initial wealth and they save through a risk-free asset with constant return. The goal is to solve the sytem of PDE's which consists of the law of motions of Euler equations. This requires initial conditions which we find in two steps. First, consider the case in which one of the households is broke. In this case we can find closedform solutions of the value functions. Second, consider the more general case in which both households have positive wealth. This yields a more general characterization of the value functions. Combining step one and two provides us with the necessary initial conditions as will become clear.

\subsection{One Household Broke}

Consider the situation in which only she has wealth and he has none. Then $\mathrm{p}=1$, and consumption of her and him are related in the following way:

$$
u^{\prime}\left(c_{t}\right)=\alpha u^{\prime}\left(c_{t}^{\prime}\right) \quad \Rightarrow \quad c_{t}^{\prime}=\alpha c_{t} .
$$

Intuitively, since he has neither income nor savings his marginal utility explodes, which induces her to transfer wealth until her marginal utility is equal to the weighted marginal utility of him. Then his consumption is proportional to hers with the proportionality factor equal to $\alpha$. Due to the homogeneity of the 
problem a reasonable guess for her consumption policy is that consumption is proportional to her wealth: $c_{t}=\bar{c} k_{t}$. With this guess of the policy compute her value of life-time utility:

$$
\begin{aligned}
V(p=1, k=K) & =\int_{0}^{\infty} e^{-\rho t}\left[\ln \left(\bar{c} k_{t}\right)+\alpha \ln \left(\alpha \bar{c} k_{t}\right)\right] d t \\
\text { subject to } & \\
\dot{k}_{t} & =r k_{t}-c_{t}-c_{t}^{\prime}=[r-(1+\alpha) \bar{c}] k_{t} .
\end{aligned}
$$

Next, we sum up savings in order to obtain an expression for wealth:

$$
\dot{k}=(r-(1+\alpha) \bar{c}) k \quad \Rightarrow \quad k_{t}=\exp \left(\int_{0}^{t}(r-(1+\alpha) \bar{c}) d w\right) k_{0},
$$

and substitute the constraint into the life-time utility:

$$
\begin{aligned}
V & =\int_{0}^{\infty} e^{-\rho t}\left\{(1+\alpha) \ln (\bar{c})+(1+\alpha)\left[\ln \left(k_{0}\right)+(r-(1+\alpha) \bar{c}) t\right]+\alpha \ln (\alpha)\right\} d t= \\
& =\frac{\alpha \ln (\alpha)}{\rho}+\frac{1+\alpha}{\rho}\left[\ln (\bar{c})+\frac{r-(1+\alpha) \bar{c}}{\rho}+\ln \left(k_{0}\right)\right] .
\end{aligned}
$$

By maximizing the expression in square brackets an expression for $\bar{c}$ is determined: $\bar{c}=\frac{\rho}{1+\alpha}$. Substituting $\bar{c}$ yields a closed-form expression for her value function:

$V\left(1, K_{0}\right)=\frac{1}{\rho}\left\{\alpha \ln (\alpha)+(1+\alpha)\left[\ln (\rho)-\ln (1+\alpha)+\left(\frac{r}{\rho}-1\right)\right]\right\}+\frac{1+\alpha}{\rho} \ln \left(K_{0}\right)$.

Her value function then takes on the simple form:

$$
V=A+B \ln \left(K_{0}\right)
$$

which confirms the guess of the consumption policy above.

On the other hand, his value function can be easily found to be:

$W\left(0, K_{0}\right)=\frac{1}{\rho}\left\{\ln (\alpha)+(1+\alpha)\left[\ln (\rho)-\ln (1+\alpha)+\left(\frac{r}{\rho}-1\right)\right]\right\}+\frac{1+\alpha}{\rho} \ln \left(K_{0}\right)$,

which takes an equally simple form as hers:

$$
W=C+B \ln \left(K_{0}\right) .
$$

The difference in the two value functions is only due to the constant term $\ln (\alpha)$ which is multiplied by $\alpha$ for agent one. Since $\alpha$ is a number between zero and one, the logarithm of it is negative, so that multiplying it by $\alpha$ increases her life-time value relative to his. 


\subsection{No Household Broke}

When $p_{t} \in[0,1]$ no closed-form solution can be found for the value functions. However, finding a general form for the value function will be useful when computing the system of PDE's. Again it is assumed that the consumption rate is independent of $\mathrm{K}: c\left(p_{t}, K_{t}\right)=\bar{c}\left(p_{t}\right) K_{t}$. Before continuing, notice that the law of motion for the wealth distribution does not depend on $K_{t}$ :

$$
\begin{aligned}
\dot{p}_{t} & =\frac{d}{d t}\left[\frac{k_{t}}{k_{t}+k_{t}^{\prime}}\right]=\frac{\dot{k}_{t}}{K_{t}}-\frac{\dot{k}_{t}+\dot{k}_{t}^{\prime}}{K_{t}} p_{t} \\
& \text { where } \\
\dot{k}_{t} & =r k_{t}-\bar{c}\left(p_{t}\right) K_{t}=r p_{t} K_{t}-\bar{c}\left(p_{t}\right) K_{t}, \\
\dot{k}_{t}^{\prime} & =r k_{t}-\bar{c}\left(1-p_{t}\right) K_{t}=r\left(1-p_{t}\right) K_{t}-\bar{c}\left(1-p_{t}\right) K_{t} \\
& \Rightarrow \dot{p}_{t}=p_{t} \bar{c}\left(1-p_{t}\right)-\left(1-p_{t}\right) \bar{c}\left(p_{t}\right) .
\end{aligned}
$$

Take a pair $\left(p_{0}, K_{0}\right)$, an initial value for the wealth distribution and total wealth in the family line, as given. Consider a set of consumption rules (we are not saying the optimal ones !) $C_{t}=\bar{c}\left(p_{t}\right) K_{t}$ for the first agent and $C_{t}^{\prime}=\bar{c}\left(1-p_{t}\right) K_{t}$ for the second. With these inputs evaluate her life-time value:

$$
\begin{aligned}
V\left(p_{0}, K_{0}\right)= & \left.\int_{0}^{\infty} e^{-\rho t}\left[\ln \left(\bar{c}\left(p_{t}\right) K_{t}\right)+\alpha \ln \left(\bar{c}\left(1-p_{t}\right) K_{t}\right)\right)\right] d t \\
& \text { subject to } \\
& \dot{K}_{t}=r K_{t}-\left(\bar{c}\left(p_{t}\right)+\bar{c}\left(1-p_{t}\right)\right) K_{t} \\
& \dot{p}_{t}=p_{t} \bar{c}\left(1-p_{t}\right)-\left(1-p_{t}\right) \bar{c}\left(p_{t}\right) .
\end{aligned}
$$

The growth rate of the combined capital stock is $\dot{K}_{t} / K_{t}=r-\left(\bar{c}\left(p_{t}\right)+\bar{c}\left(1-p_{t}\right)\right)$ so that the logarithm of the capital stock at time $t$ is given by:

$$
\begin{aligned}
& K_{t}=\exp \left(\int_{0}^{t}\left[r-\left(\bar{c}\left(p_{\tau}\right)+\bar{c}\left(1-p_{\tau}\right)\right] d \tau\right) K_{0}\right. \\
& \Rightarrow \ln \left(K_{t}\right)=\ln \left(K_{0}\right)+r t-\int_{0}^{t}\left[\bar{c}\left(p_{\tau}\right)+\bar{c}\left(1-p_{\tau}\right)\right] d \tau
\end{aligned}
$$

Integrating this expression:

$$
\begin{aligned}
& \Rightarrow \int_{0}^{\infty} e^{-\rho t}\left\{\ln \left(K_{0}\right)+r t-\int_{0}^{t}\left[\bar{c}\left(p_{\tau}\right)+\bar{c}\left(1-p_{\tau}\right)\right] d \tau\right\} d t= \\
& =\frac{\ln \left(K_{0}\right)}{\rho}+\frac{r}{\rho^{2}}+\int_{0}^{\infty} e^{-\rho t}\left\{-\int_{0}^{t}\left[\bar{c}\left(p_{\tau}\right)+\bar{c}\left(1-p_{\tau}\right)\right] d \tau\right\} d t
\end{aligned}
$$


Life-time value for the agent is then given by:

$$
\begin{aligned}
& V\left(p_{0}, K_{0}\right)=\frac{1+\alpha}{\rho}\left[\frac{r}{\rho}+\ln \left(K_{0}\right)\right]+ \\
&+\int_{0}^{\infty} e^{-\rho t}\left\{\ln \left(\bar{c}_{t}\right)+\alpha \ln \left(\bar{c}_{t}\left(1-p_{t}\right)\right)-(1+\alpha) \int_{0}^{t}\left[\bar{c}\left(p_{\tau}\right)+\bar{c}\left(1-p_{\tau}\right)\right] d \tau\right\} d t
\end{aligned}
$$

where we have shown above that $p_{t}$ does not depend on $K_{t}$ and therefore we don't bother substituting it out by integrating its law of motion. Thus, the integral is only a function of $p_{0}$. We call this function $v$ and write for a given $\left(p_{0}, K_{0}\right)$ :

$$
\begin{aligned}
V\left(p_{0}, K_{0}\right) & =\frac{1+\alpha}{\rho}\left[\frac{r}{\rho}+\ln \left(K_{0}\right)\right]+v\left(p_{0}\right) \\
& \text { subject to } \\
& \dot{p}_{t}=p_{t} \bar{c}\left(1-p_{t}\right)-\left(1-p_{t}\right) \bar{c}\left(p_{t}\right)
\end{aligned}
$$

Why do we need this expression? It characterizes how life-time utility depends on wealth and the distribution of wealth. It allows us to study small changes in value with changes in $\mathrm{p}$. In the case we considered above, in which one of the agents is broke this is impossible, since by definition $\mathrm{p}$ does not change (in the case wehn one of the agents is broke $\mathrm{p}=1$ by definition).

What we are after are, of course, optimal consumption policies. In order for this policy to be optimal it needs to satisfy the Hamilton-Jacobi-Bellman equation. The HJB tells us that we need a relationship between optimal consumption today and tomorrow which is related to the marginal value of wealth accumulation. As above differentiate the HJB w.r.t. $k, k^{\prime}$, and similarly for the other agent. This gives the system of PDE's to be solved.

How do we solve this system of PDE's? Tedious algebra reveals that the system of PDE's simplifies to a system of ODE's. As such, two boundary conditions are required. Here we will make use of the case when she is broke, namely, when $\mathrm{p}=0$. Define $T$ to be the time in which she becomes optimally bankrupt. From the case studied above we know that for $t \geq T$ she consumes:

$$
C\left(K_{0}^{\prime}\right)=\alpha\left(\frac{\rho}{1+\alpha} K_{0}^{\prime}\right), \quad K_{0}^{\prime}=k_{0}^{\prime}
$$

and her marginal utility is given by

$$
\frac{1}{C_{0}}=\frac{1}{\alpha}\left(\frac{1+\alpha}{\rho}\right) \frac{1}{K_{0}^{\prime}}
$$

For $t<T$ the she is unconstrained and we can read of optimal consumption from her FOC:

$$
V_{k}\left(p_{0}, K_{0}\right)=\frac{d V}{d p_{0}} \frac{d p_{0}}{d k_{0}}+\frac{d V}{K_{0}} \frac{d K_{0}}{d k_{0}}=\frac{1}{K_{0}}\left(\frac{1+\alpha}{\rho}+v^{\prime}\left(p_{0}\right)\right)=u^{\prime}\left(C\left(p_{0}, K_{0}\right)\right)
$$


In order to find her consumption in the moment before she is about to become broke we make use of her HJB:

$$
\left\langle V_{k}^{l i m}, V_{k^{\prime}}\right\rangle \cdot\left\langle c_{0}^{l i m},-\dot{k}^{\prime l i m}\right\rangle=u\left(c_{0}^{l i m}\right)+\alpha u\left(c_{1}^{\prime}\right)-\rho V
$$

Similarly, his HJB tells us:

$$
\left\langle W_{k}, W_{k^{\prime}}\right\rangle \cdot\left\langle c_{0}^{l i m},-\dot{k}^{\prime l i m}\right\rangle=u\left(c_{1}^{\prime}\right)+\alpha u\left(c_{0}^{l i m}\right)-\rho W .
$$

With the first condition we can back out $c_{0}^{\text {lim }}$ - her limiting consumption - and with the second $W_{k}$ - his cross-derivative. This information enables us to back out $v^{\prime}(0)$ and $w^{\prime}(0)$. The elements of the first PDE are:

$$
\begin{aligned}
V_{k}^{l i m} & \equiv \lim _{\epsilon \rightarrow 0} V_{k}(\epsilon, K)=u^{\prime}\left(c_{0}^{l i m}\right) \\
V_{k^{\prime}} & \equiv V_{k^{\prime}}\left(0, K^{\prime}\right)=\frac{1+\alpha}{\rho} \frac{1}{K^{\prime}} \\
c_{1}^{\prime} & \equiv \frac{\rho}{1+\alpha} K^{\prime} \\
\dot{k}^{\prime} l i m & \equiv r K^{\prime}-c_{1}^{\prime}
\end{aligned}
$$

Equation (13) can be solved for $V_{k}^{\text {lim }}$ using (16)- (18). Combining this with equation (15) yields two equations in two unknowns $V_{k}^{\text {lim }}$ and $c_{0}^{\text {lim }}$. Then we can solve for her limiting consumption:

$$
c_{0}^{l i m}=c_{0} \exp (1-\alpha), \quad c_{0}=\alpha \frac{\rho}{1+\alpha} K^{\prime} .
$$

Now we can use the fact that we have a general form of the value function which enables us to back out $v^{\prime}(0)$ :

$$
\begin{aligned}
\frac{1}{c_{0}^{l i m}} & =\left.V_{k}\right|_{p=0}=\frac{1}{K^{\prime}}\left(\frac{1+\alpha}{\rho}+v^{\prime}(0)\right) \\
& \Rightarrow v^{\prime}(0)=\frac{1+\alpha}{\rho}\left(\frac{\exp (\alpha-1)-\alpha}{\alpha}\right)
\end{aligned}
$$

For the second PDE we note that:

$$
W_{k^{\prime}} \equiv \frac{1+\alpha}{\rho} \frac{1}{K^{\prime}}
$$

all the other elements that we require to solve for $W_{k}$ we already know. From equation (14) we get that:

$$
W_{k}=(2-\alpha) \exp (\alpha-1) \frac{1}{c_{1}}, \quad c_{1}=\frac{\alpha}{1+\rho} K^{\prime} .
$$

Again, from our general value function we have:

$$
\left.W_{k}\right|_{p=0}=\frac{1}{K^{\prime}}\left(\frac{1+\alpha}{\rho}+w^{\prime}(0)\right)
$$

which enabless us to back out the second initial condition:

$$
w^{\prime}(0)=\frac{1+\alpha}{\rho}((2-\alpha) \exp (\alpha-1)-1) .
$$




\title{
6 An example with uncertainty in income
}

\author{
TO BE WRITTEN
}

\section{Conclusions}

This paper provides a dynamic model of voluntary transfers with two-sided altruism in a Markov-perfect setting. The continuous-time setting allows us to neglect certain second-order effects and provides additional tractability.

We find that transfers are especially likely when the recipient is liquidityconstrained, which is in line with results from the empirical literature. We show that the equilibrium allocation is not Pareto-efficient because agents' savings decisions are distorted by altruistic-strategic considerations. Agents have an extra disincentive to save since they fear that their relatives react by overspending in reliance on their larger resources.

A finding that distinguishes our model from the static setting of altruism is that donors condition transfers on assets and income in distinct ways. Furthermore, the model has predictions on savings decisions that we plan to test in future versions of the paper. We also plan to assess the quantitative implications of our theory in computational examples.

In future work, we plan to use our model as a building block for a dynamic theory on long-term-care decisions in order to evaluate different policy proposals.

\section{References}

Altonji, J. G., Hayashi, F. \& Kotlikoff, L. J. (1997), 'Parental altruism and inter vivos transfers: Theory and evidence', Journal of Political Economy 105, 1121-1166.

Barro, R. J. (1974), 'Are government bonds net wealth?', The Journal of Political Economy 82, 1095-1117.

Becker, G. S. (1974), 'A theory of social interactions', The Journal of Political Economy 82, 1063-1093.

Berry, B. (2008), 'Financial transfers from living parents to adult children: Who is helped and why?', American Journal of Economics and Sociology 67, 207-239.

Brown, M. (2007), End-of-life transfers and the decision to care for a parent. Working Paper, University of Wisconsin.

Cox, D. (1987), 'Motives for private income transfers', The Journal of Political Economy 95, 508.

Cox, D. (1990), 'Intergenerational transfers and liquidity constraints', Quarterly Journal of Economics 105, 187-217. 
Cox, D. \& Jappelli, T. (1990), 'Credit rationing and private transfers: Evidence from survey data', Review of Economics and Statistics 72, 445-454.

Cox, D. \& Raines, F. (1985), Interfamily transfers and income redistribution. Working Paper.

Fernandes, A. (2008), A closed-form solution to a model of two-sided, partial altruism. Working Paper.

Gale, W. G. \& Scholz, J. K. (1994), 'Intergenerational transfers and the accumulation of wealth', Journal of Economic Perspectives 8, 145-160.

Kocherlakota, N. R. (1996), 'Implications of efficient risk sharing without commitment', Review of Economic Studies 63, 595-609.

Laferrere, A. \& Wolff, F. C. (2006), 'Microeconomic models of family transfers', Handbook of the Economics on Giving, Altruism and Reciprocity 2, 889969.

Laitner, J. (1988), 'Bequests, gifts, and social security', The Review of Economic Studies 55, 275-299.

Laitner, J. (1997), 'Intergenerational and interhousehold economic links', Handbook of Population and Family Economics 1, 189-238.

Laitner, J. P. (1992), 'Random earnings differences, lifetime liquidity constraints, and altruistic intergenerational transfers', Journal of Economic Theory 58, 135-170.

McGarry, K. (2006), Testing parental altruism: Implications of a dynamic model. Working Paper.

McGarry, K. \& Schoeni, R. F. (1995), Transfer behavior within the family: Results from the asset and health dynamics survey. Working Paper.

Soldo, B. J. \& Hill, M. S. (1995), 'Family structure and transfer measures in the health and retirement study: Background and overview', Journal of Human Resources 30, 108-137.

Townsend, R. (1979), 'Optimal contracts and competitive markets with costly state verification', Journal of Economic Theory 21, 265-293.

\section{A Proof: Impulse response for consumption}

Claim: If consumption reacts positively on either capital stock (i.e. $c_{k}\left(k, k^{\prime}\right) \geq$ $0, c_{k^{\prime}}\left(k, k^{\prime}\right) \geq 0$ for all $\left(k, k^{\prime}\right)$ - and the equivalent expression for $c^{\prime}-$, then a small positive change in $k_{0}$ will always entail non-negative changes in $k_{t}$ for all $t \geq 0)$ and non-positive changes in $k_{t}^{\prime}$.

Proof: Fix an equilibrium path $\left(k, k^{\prime}\right)$ for the system starting at $\left(k_{0}, k_{0}^{\prime}\right)$. When changing her capital stock at $t=0$ upward a bit by some amount $h_{0}$, we 
are looking for the equilibrium trajectory of the system that this entails, i.e. we want functions in time $\left(k_{t}+h_{t}, k_{t}^{\prime}+h_{t}^{\prime}\right)$ where we have deviations $\left(h_{t}, h_{t}^{\prime}\right)$ from equilibrium. This means that for any $t<0$ we must have:

$$
\begin{array}{r}
\left(k_{t}+\epsilon h_{t}\right)-\left(k_{0}+\epsilon h_{0}\right)-\int_{0}^{t} y+r\left(k_{s}+\epsilon h_{s}\right)-c\left(k_{s}+\epsilon h_{s}, k_{s}^{\prime}+\epsilon h_{s}^{\prime}\right) d s=0 \\
\left(k_{t}^{\prime}+\epsilon h_{t}^{\prime}\right)-\left(k_{0}^{\prime}+\epsilon h_{0}^{\prime}\right)-\int_{0}^{t} y^{\prime}+r\left(k_{s}^{\prime}+\epsilon h_{s}^{\prime}\right)-c^{\prime}\left(k_{s}+\epsilon h_{s}, k_{s}^{\prime}+\epsilon h_{s}^{\prime}\right) d s=0
\end{array}
$$

Differentiate with respect to $\epsilon$ at $\epsilon=0$ to obtain

$$
\begin{aligned}
& h_{t}-h_{0}-\int_{0}^{t} r h_{s}-c_{k}\left(k_{s}, k_{s}^{\prime}\right) h_{s}-c_{k^{\prime}}\left(k_{s}, k_{s}^{\prime}\right) h_{s}^{\prime} d s=0 \\
& h_{t}^{\prime}-h_{0}^{\prime}-\int_{0}^{t} r h_{s}^{\prime}-c_{k}^{\prime}\left(k_{s}, k_{s}^{\prime}\right) h_{s}-c_{k^{\prime}}^{\prime}\left(k_{s}, k_{s}^{\prime}\right) h_{s}^{\prime} d s=0
\end{aligned}
$$

Now, differentiate both equations with respect to $t$ to obtain a system of ordinary differential equations (ODEs) for $h$ and $h^{\prime}$ :

$$
\begin{aligned}
& \dot{h}_{t}=\left[r-c_{k}\left(k_{t}, k_{t}^{\prime}\right)\right] h_{t}-c_{k^{\prime}}\left(k_{t}, k_{t}^{\prime}\right) h_{t}^{\prime} \\
& \dot{h}_{t}^{\prime}=\left[r-c_{k}^{\prime}\left(k_{t}, k_{t}^{\prime}\right)\right] h_{t}^{\prime}-c_{k}\left(k_{t}, k_{t}^{\prime}\right) h_{t}
\end{aligned}
$$

Now, we will choose $h_{0}=1$ and $h_{0}^{\prime}=0$ to see what this implies for the impulse response. For small $t$, we will have $\dot{h}_{t}^{\prime}<0$ since we assumed $c_{k}>0$ and $h_{t}^{\prime}$ is still very small compared to $h_{t} \simeq 1$. Now, we will argue that the system cannot escape from a region where $h>0$ and $h^{\prime}<0$, which means that the impulse responses maintain the same sign forever.

If the system indeed escaped from the region where $h>0$ and $h^{\prime}<0$, then there must be some $T$ where this happens, which we define as

$$
T \equiv \inf _{t \geq 0}\left\{t \mid h_{t}<0 \text { or } h_{t}^{\prime}>0\right\}
$$

Notice that by continuity of $h$ and $h^{\prime}$ we have either $h_{T}=0$ or $h_{T}^{\prime}=0$ (or both). First, suppose that $h_{t}$ crossed zero at $T$ but $h_{T}^{\prime} \leq 0$. Then we must have $\dot{h}_{T}=-c_{k^{\prime}}\left(k_{T}, k_{T}^{\prime}\right) h_{T}^{\prime}>0$, which contradicts the assumption that $h_{T}^{\prime} \leq 0$. Second, it might be that $h_{t}^{\prime}$ became positive at some point $T$ and we had $h_{T}^{\prime} \geq 0$ still. This would imply $\dot{h}_{T}^{\prime}=-c_{k}\left(k_{T}, k_{T}^{\prime}\right) h_{T} \leq 0$, which contradicts the fact that $\dot{h}$ becomes positive after $T$.

But this does not imply that we know the sign of the impulse response for $c$; we have

$$
\frac{d c_{t}}{d k_{0}}=c_{k}\left(k_{t}, k_{t}^{\prime}\right) \frac{d k_{t}}{d k_{0}}+c_{k^{\prime}}\left(k_{t}, k_{t}^{\prime}\right) \frac{d k_{t}^{\prime}}{d k_{0}}
$$

whose sign is not determined by the above lemma. 